Article

\title{
Fine-Grained Power Gating Using an MRAM-CMOS Non-Volatile Flip-Flop
}

\author{
Jaeyoung Park * (iD) and Young Uk Yim \\ School of Computer Science and Electrical Engineering, Handong Global University, Pohang-si 37554, Korea; \\ zero12@gmail.com \\ * Correspondence: jaeyoung.park@handong.edu; Tel.: +1-858-658-2657
}

Received: 28 April 2019; Accepted: 17 June 2019; Published: 20 June 2019

\begin{abstract}
An area-efficient non-volatile flip flop (NVFF) is proposed. Two minimum-sized MetalOxide-Semiconductor Field-Effect Transistor (MOSFET) and two magnetic tunnel junction (MTJ) devices are added on top of a conventional $\mathrm{D}$ flip-flop for temporary storage during the power-down. An area overhead of the temporary storage is minimized by reusing a part of the D flip-flop and an energy overhead is reduced by a current-reuse technique. In addition, two optimization strategies of the use of the proposed NVFF are proposed: (1) A module-based placement in a design phase for minimizing the area overhead; and (2) a dynamic write pulse modulation at runtime for reducing the energy overhead. We evaluated the proposed NVFF circuit using a compact MTJ model targeting an implementation in a $10 \mathrm{~nm}$ technology node. Results indicate that area overhead is $6.9 \%$ normalized to the conventional flip flop. Compared to the best previously known NVFFs, the proposed circuit succeeded in reducing the area by $4.1 \times$ and the energy by $1.5 \times$. The proposed placement strategy of the NVFF shows an improvement of nearly a factor of 2-18 in terms of area and energy, and the pulse duration modulation provides a further energy reduction depending on fault tolerance of programs.
\end{abstract}

Keywords: STT-MRAM; flip-flop; power gating; low-power

\section{Introduction}

Power gating has been researched as an effective energy-reduction technique [1-3]. This reduces static power consumption by shutting power off. However, data needs to be transferred to another storage component before the power-off and restored after the power-on [4,5]. Such transfers of data introduce energy and area overheads. Therefore, it is important to develop a low overhead temporary storage component and an efficient strategy of the use of the storage components. Off-chip memories have been used for the temporary storage [2,3]; however, a complex interface between the off-chip memories and a chip stands in the way of wide adoption of such off-chip memories in power gating scheme.

Embedded non-volatile flip-flops (NVFFs) are promising enablers to fine-grained power gating because these do not require a complex interface to transfer state from/to the external storage. One critical issue is an overhead to store/restore data onto non-volatile temporary storage of the NVFF before/after the power-down. It is universally the case when adding a new feature (e.g., non-volatility) to the existing flip-flop. However, what is the best way to build a low overhead NVFF? A magnetic tunnel junction (MTJ) of a spin torque transfer magnetic memory (STT-MRAM) is a candidate for the non-volatile storage of the NVFF because the MTJ does not occupy the silicon area; the MTJ is placed between metal layers. However, the area and energy overheads can be significant if write and read circuits for the MTJ are not carefully optimized. 
In this paper, we propose an area-efficient MRAM-Complementary Metal-Oxide-Semiconductor (CMOS) hybrid non-volatile flip-flop. We reutilize the existing CMOS flip-flop for transferring data to/from MTJs to reduce the area overhead. Only two minimum-sized transistors are added. In addition, the energy overhead for storing data onto MTJs is reduced by $50 \%$ by reusing a write current to write two MTJs. We evaluated the proposed NVFF circuit using a compact MTJ model and a 10 nm Predictive Technology Model (PTM) MOSFET model [6-8]. The proposed NVFF has an improvement by a factor of 4-23 in terms of the area over state-of-the-art circuits. In addition, energy for the storing operation is reduced by $1.5 \times$ compared to the best previously proposed NVFF circuits.

We also propose optimization strategies for the use of the proposed NVFF. Because the proposed NVFF introduces a non-negligible area overhead compared to the conventional D flip-flop (FF), it is important to use the proposed NVFF carefully to minimize the area overhead. Replacing all the conventional FFs in a design with NVFFs imposes a large area penalty. Therefore, we first analyze a circuit and then place the NVFFs only for a selected module which can minimize the area penalty. We place the proposed NVFF in a module that has a low ratio of the flop area to total. Because a module contains flops, combinational logic circuits, and passive devices, the area penalty by the NVFF is minimized where FFs occupy the relatively small area to the total. In the other words, the area increase by the NVFF becomes relatively small if the other components in the same modules occupy the greater portion of the module.

In addition, the write pulse duration for the NVFF needs to be carefully optimized due to the stochastic nature of the MTJ write. The MTJ write process itself is fundamentally stochastic and the actual time to the completion varies dramatically with the distribution having a very long tail [9-11]. This means that write energy also varies quite significantly and the write energy can be wasteful if the applied write pulse duration is not carefully selected. Instead of using the conventional deterministic strategy with a fixed pulse duration that guarantee a target error probability, we exploit this stochastic property to save more energy by adjusting the pulse duration adaptively. A key insight is that high fault-tolerance programs can endure more error from the NVFF so that we can reduce the write pulse duration for the programs to save write energy even if the NVFF itself introduces higher error probability.

We demonstrate the optimization strategies on an OpenSPARC design which is an open-source version of UltraSPARC processor [12]. Four programs-matrix multiply, sort, bzip2, and prime-are also selected for this experiment [13]. Analysis indicates the placement shows an improvement of nearly a factor of 2-18 in terms of area and energy and the pulse duration modulation maximizes energy savings of the proposed NVFF for programs have high fault tolerance. The detailed analysis are presented in the following sections.

\section{MRAM-CMOS Non-Volatile Flip-Flops}

\subsection{State-of-the-Art MRAM-CMOS NVFFs}

MRAM-CMOS NVFFs typically need extra circuits for writing and reading MTJs. Multiple realizations of the extra circuits, which use additional write drivers and sense amplifiers, have been proposed [14-17]. In [15], two NAND gates, seven inverters, and three NMOS switch transistors are used for the external write driver and the sense amplifier with a significant reduction of D-Q delay. In [16], four NOR gates, four inverters, and 16 NMOS transistors are used to reduce C-Q delay and sensing currents.

In [14], only three extra transistors are added for writing and reading MTJs because the existing cross-coupled inverter pair of the conventional D-FFs is used to assist with storing and restoring operations of MTJs. Figure 1 shows the storing and restoring operations of the NVFF [14]. For the storing operation, $M T J_{A}$ is written to antiparallel (AP) state by lowering Reset-ENable (REN) signal when $\mathrm{QS}$ is logical ' $\mathrm{H}$ ' for $\mathrm{Q}=1 . M T J_{B}$ is written to parallel $(\mathrm{P})$ state by raising the $\mathrm{REN}$ and Set-ENable (SEN) signals in the second write phase. The restoring operation is achieved by the regenerative feedback of the inverter pair because a different voltage is developed between QS and QSb nodes 
when $M T J_{A}$ and $M T J_{B}$ have different resistances. This is why the NVFF requires only three additional transistors. However, sizable transistors are needed to drive sufficient current with low Vgs because the Vgs is dropped by IR drop through an MTJ (Vgs $\left.=\mathrm{Vdd}-\mathrm{I} \times R_{M T J}\right)$. Moreover, the storing energy is doubled because two MTJs need to be written in different phases.

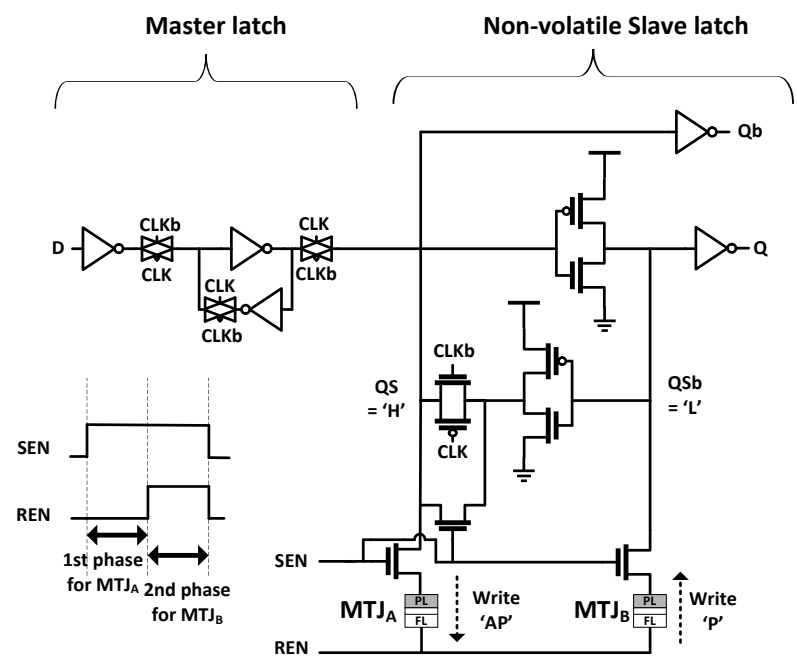

(a) Storing operation $(\mathrm{Q}=1)$

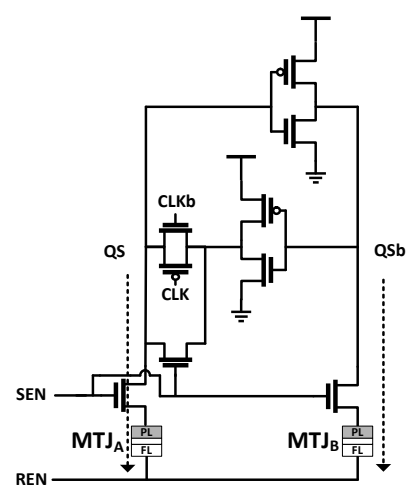

(b) Restoring operation (Slave latch only)

Figure 1. Schematic of an non-volatile flip flop (NVFF) of Yamamoto et al. [14].

\subsection{Current Reutilization NVFF}

We propose a current reutilization technique to reduce energy and area overheads. The main idea that a single write current for an MTJ can be used to write another MTJ. Instead of applying two separate current pulses to write two MTJs at different phases, we can write two MTJs using a single write current at the same time. The current reutilization should not introduce large area overhead. We achieved this by inserting one minimum-sized NMOS transistor because two MTJs can be placed on the same current path via the NMOS transistor (M1) as shown in Figure 2. A write current is passed through $M T J_{A}, \mathrm{M} 1$, and $M T J_{B}$ when a switch transistor (M1) is turned on for $\mathrm{Q}=1$. The $M T J_{A}$ is written to the AP state because the current direction is from the pinned layer (PL) to the free layer $(\mathrm{FL})$ of the MTJ, and $M T J_{B}$ becomes the P state because the current direction is reversed (FL $\rightarrow$ PL). For storing $\mathrm{Q}=0$, a write current goes through $M T J_{B}, \mathrm{M} 1$, and $M T J_{A}$; therefore, the situation is reversed $\left(M T J_{B}=\mathrm{AP}, M T J_{A}=\mathrm{P}\right)$. The proposed current reutilization technique allows for writing both MTJs using one write current at the same time. Thus, we could reduce the write current by $50 \%$, resulting in a half storing energy. In contrast to an NVFF of Yamamoto et al. [14], an inverter pair drives a write current and a minimum-sized NMOS transistor is only used as a switch. In addition, a full Vdd is applied to a gate of the inverter pair during the storing operation.

The restoring operation is achieved by another minimum-sized transistor (M2). This reutilizes the inverter pair of the existing D-FF. Different voltages are developed between QS and QSb of the slave latch by two MTJs that have different resistances when M1 and M2 are turned on after the power-up. 


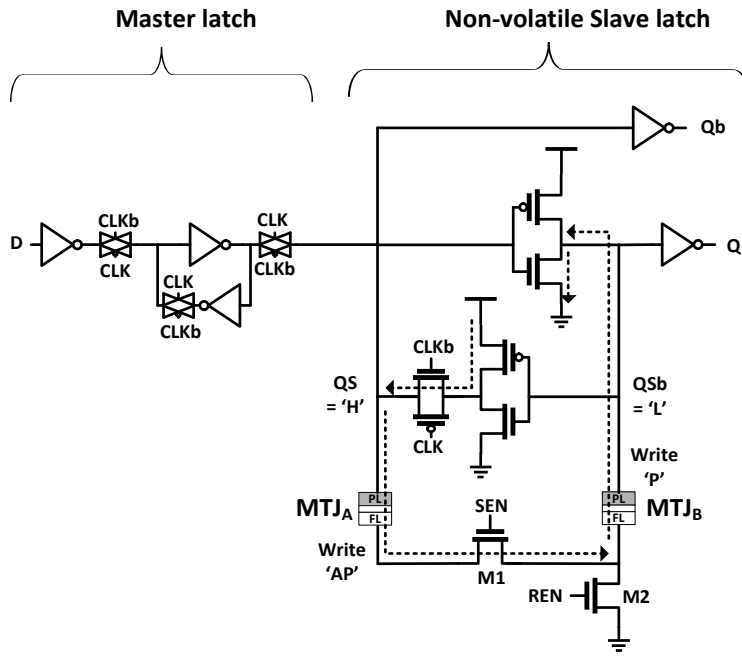

(a) Storing operation $(\mathrm{Q}=1)$

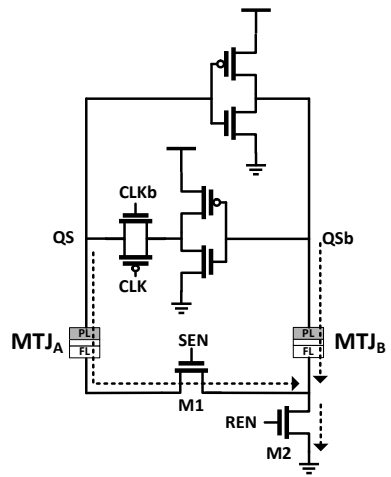

(b) Restoring operation (Slave latch)

Figure 2. Schematic of the proposed current reutilization NVFF.

\subsection{Evaluation of the Proposed NVFF}

We designed the proposed NVFF using a $10 \mathrm{~nm}$ predictive technology model (PTM) MOSFET model and a compact MTJ model [7,8]. Key parameters of the perpendicular MTJ is described in Table 1. Figure 3 shows Simulation Program with Integrated Circuit Emphasis (SPICE) simulation results of the proposed NVFF using the models. The proposed NVFF operates as a conventional D-FF in normal operations. On top of the D-FF, non-volatile operations are added. The storing operation is performed before the power-down. The output $\mathrm{Q}$ is stored in MTJs when SEN is raised. $M T J_{A}$ is written to the $\mathrm{AP}$ state and $M T J_{B}$ is the $\mathrm{P}$ state for $\mathrm{Q}=1$. During the power-down mode, the output $\mathrm{Q}$ is lowered. The $\mathrm{Q}$ is restored when power is up again at $28.6 \mathrm{~ns}$ (restoring operation).

Table 1. Key parameters of perpendicular magnetic tunnel junction (MTJ) [6-8].

\begin{tabular}{ccc}
\hline Parameter & Value & Unit \\
\hline Intrinsic critical current & 24 & $\mu \mathrm{A}$ \\
Thermal stability factor & 58 & \\
Tunnel Magnetoresistance ratio (TMR) & $\sim 100$ & $\%$ \\
Diameter of MTJ & 20 & $\mathrm{~nm}$ \\
Out-of-plane magnetic field & 0.4 & $\mathrm{~T}$ \\
\hline
\end{tabular}

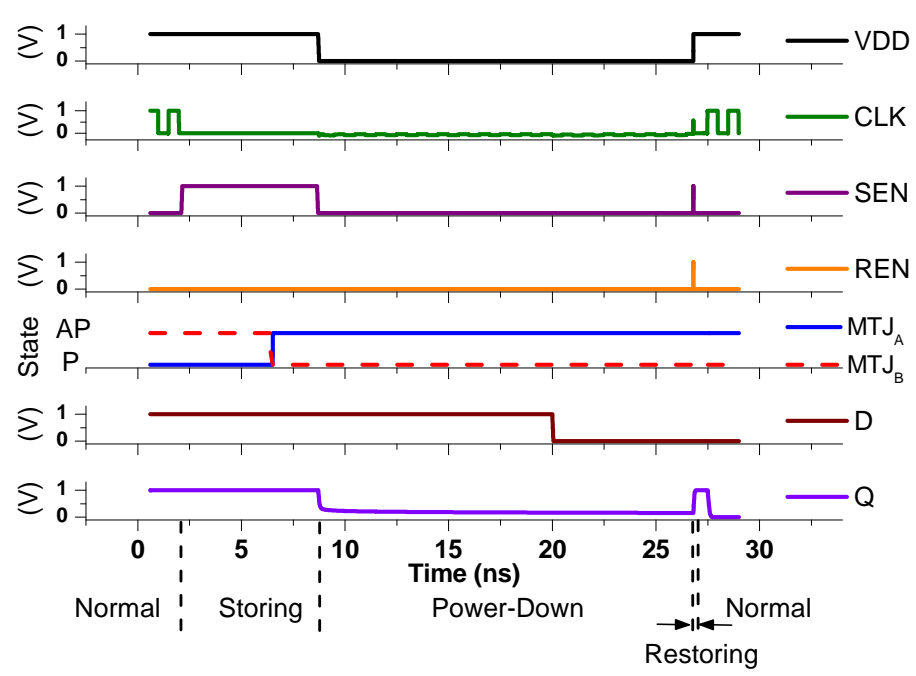

Figure 3. Waveforms of each node of the proposed NVFF. (Output ' 1 ' is stored and restored. $x$-axis denotes time and the $y$-axes indicates voltage (V) or states of MTJs.) 
We compare the proposed NVFF with the state-of-the-art NVFF circuits as shown in Table 2. The proposed NVFF shows an improvement of nearly a factor of 2-17 in terms of restoring energy compared to the state-of-the-art NVFF circuits. Note that the restoring energy is reduced by $50 \%$ if MTJ and CMOS devices are the same as an NVFF [14]. The proposed NVFF implemented in an advance technology node and the greater part of the storing energy reduction comes from the technology scaling.

The relative area increase is only $6.9 \%(2 / 29)$ because only two minimum-sized transistors are added to the conventional D flip-flop (FF) that has 29 transistors. We did not directly compare the area because technologies of the reference circuits are different, and the actual area strongly depends on the layout optimization. Thus, we used a relative area overhead to the D-FF of each technology for this comparison. Note that the size of the PMOS transistor in the inverter is assumed to be $2 \times$ NMOS transistor. The relative area overheads of state-of-the-art NVFF architectures are from $28.0 \%$ to $160.0 \%$. Therefore, the proposed NVFF has an improvement of nearly a factor of 4-23 in terms of the area overhead compared to state-of-the-art NVFF circuit.

Table 2. Performance summary and comparison with the state-of-the-art NVFFs.

\begin{tabular}{|c|c|c|c|c|c|c|c|c|c|c|}
\hline & & \multicolumn{7}{|c|}{ MRAM-Based } & \multirow{2}{*}{$\frac{\text { FeRAM- }}{[20]}$} & \multirow{2}{*}{$\frac{\text { ReRAM- }}{[21]}$} \\
\hline & & This Work & [17] & [16] & [15] & [18] & [14] & {$[19]^{a}$} & & \\
\hline \multicolumn{2}{|c|}{ Technology node (nm) } & 10 & 90 & 45 & 45 & 90 & 65 & 65 & 130 & 65 \\
\hline \multicolumn{2}{|c|}{ Area overhead ${ }^{b}(\%)$} & 6.9 & 131.0 & 160.0 & 120.0 & 103.4 & 109.0 & 28.0 & 64.0 & 32.0 \\
\hline \multirow{2}{*}{ Energy $(\mathrm{pJ})$} & Storing & 0.2 & 175.5 & 1.9 & 1.6 & 0.3 & 5.0 & 0.5 & 2.4 & - \\
\hline & Restoring & 0.002 & - & 0.171 & 0.007 & - & 0.349 & 0.197 & - & - \\
\hline \multirow{2}{*}{ Delay (ns) } & Storing & 6.6 & - & - & - & 10.0 & 29.5 & 6.4 & 1640.0 & - \\
\hline & Restoring & 0.01 & $0.169^{c}$ & 2.0 & 0.184 & 1.0 & 2.0 & 2.0 & 1230.0 & 16.0 \\
\hline \multicolumn{2}{|c|}{ C-Q delay (ps) } & 43.8 & $318.1^{\mathrm{c}}$ & 68.8 & 186.2 & 67.2 & 73.8 & - & - & $<1 \mathrm{~ns}$ \\
\hline \multicolumn{2}{|c|}{ Power-Delay Product (fJ) } & 0.3 & $2.8^{c}$ & 1.1 & 2.3 & 0.7 & 1.4 & - & - & - \\
\hline
\end{tabular}

A simulated delay of the restoring operation is $10 \mathrm{ps}$ and a storing time is set to $6.6 \mathrm{~ns}$ to have a sufficiently low error probability. We computed the error probability of the proposed NVFF. We used the following probability model derived in [11] using a Neel-Brown relaxation formula to compute error probability. The model describes the switching probability $P_{S W}(t, I)$, which is the probability of switching occurring for a pulse duration $t$ at current $I$ :

$$
P_{S W}(t, I)=1-e^{-\frac{t}{\tau_{0} e^{\Delta\left(1-I / I_{c 0}\right)}}}
$$

where $\Delta$ is the thermal stability factor and $\tau_{0}$ is is the inverse of the thermal attempt frequency that has a typical value of $1 \mathrm{~ns}[10,11]$. $I_{C 0}$ is a critical current and $I$ is an applied current to write. A computed write error probability is $1.5 \times 10^{-13}$ where an average write current is $24.6 \mu \mathrm{A}$ and a storing time is $6.6 \mathrm{~ns}$.

\section{Optimization Strategies for the Proposed NVFF}

In this section, we describe optimization strategies of the use of the proposed NVFF. Because the proposed NVFF introduces a non-negligible area overhead compared to the conventional D-FF, it is important to use the proposed NVFF carefully to minimize the area overhead. In addition, the MTJ write process itself is fundamentally stochastic and the actual time to completion varies dramatically with the distribution having a very long tail [9-11]. This means that write energy also varies quite significantly and the write energy can be wasted if the applied pulse duration is not carefully adjusted. 
We propose a two-phase optimization strategy: (1) a static NVFF placement in a design phase and (2) dynamic pulse width modification at runtime. The proposed two-phase flow is illustrated in Figure 4. In a design phase, we place the NVFF only in a module that is able to maximize the benefit of the NVFF. At runtime, we dynamically adjust the write pulse duration to save more energy for a program that has high fault tolerance. The details are described as follows.

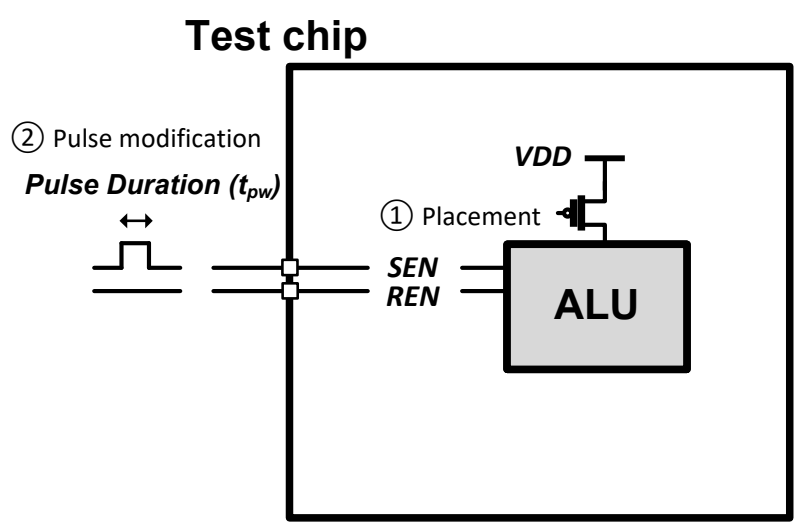

Figure 4. Overview of the proposed two-phase optimization flow.

\subsection{Pre-Fabrication Optimization: A Module-Based Placement}

A key question for the optimization is where the NVFF is placed to reduce static power while minimizing the area overhead. Replacing all the conventional FFs with NVFFs imposes a large area penalty. Therefore, we first analyze a circuit and then place the NVFFs only for a selected module. This is a fine-grained (or cluster-based) power gating approach. We characterize a circuit using two metrics, static power and a ratio of the FF area to the total, and then place the NVFFs in a module that has high static power and low area ratio. Because the area penalty is minimized where FFs occupy the relatively small area to the total. In addition, more static power can be saved if the module itself consumes high static power. Because the power gating can reduce static (leakage) power by shutting off power supply and it has no impact on dynamic power, placing the NVFFs in a high-static-power module can save more static power. Because a module generally contains not only FFs but also has combinational logic circuits and passive devices, the power gating can also reduce the static power of the combinational logic circuits and passive devices in the same module too.

We demonstrate the proposed optimization strategy in designing OpenSPARC T1 core, which is an open-source version of UltraSPARC processor. We first synthesized all modules and performed the placement and routing using Synopsys $32 \mathrm{~nm}$ EDK standard cell library [23]. We used Synopsys Design Compiler, IC Compiler, and Primetime for synthesizing, placement and routing, and static timing and power analysis, respectively [24-26]. We then selected seven high computational modules, and analyzed area and static power. As shown in Table 3, ALU (exu_alu) and decoder (ifu_dec) modules have fewer FFs than the other five modules. This results in lower area ratio to total. The increased area is less than $1 \%$ if the conventional FFs in the modules are replaced with the proposed NVFFs. Among two modules, the static power of the ALU is higher than that of the decoder. Therefore, we selected the ALU for a module to place the proposed NVFF. The placement shows an improvement of nearly a factor of 2-18 in terms of area and energy compared to the other modules.

All performances of seven modules are summarized in Table 3. The area and power are computed using Synopsys Primetime. Storing and restoring energy from the $10 \mathrm{~nm}$ PTM model are scaled up based on a constant field scaling method [27] because $32 \mathrm{~nm}$ standard cell library is used for the placement and routing of the OpenSPARC core. A break-even time $\left(T_{\text {breakeven }}\right)$ is determined when energy saving by the power-gating is equal to the energy overhead by storing and restoring operations. 
Table 3. Performance summary of seven modules.

\begin{tabular}{ccccccccc}
\hline & $\begin{array}{c}\text { FF } \\
\text { Area }\left(\mu \mathbf{m}^{\mathbf{2}}\right)\end{array}$ & $\begin{array}{c}\text { Total } \\
\text { Area }\left(\mu \mathbf{m}^{\mathbf{2}}\right)\end{array}$ & $\begin{array}{c}\text { FF/Total } \\
(\mathbf{\%})\end{array}$ & $\begin{array}{c}\text { NVFF } \\
\text { Area }\left(\mu \mathbf{M}^{\mathbf{2}}\right)\end{array}$ & $\begin{array}{c}\text { Increased } \\
\text { Area }(\mathbf{\%})\end{array}$ & $\begin{array}{c}\boldsymbol{P}_{\text {static }} \\
(\mathbf{m W})\end{array}$ & $\begin{array}{c}\boldsymbol{E}_{\text {storing }} \\
+E_{\text {restoring }}(\mathbf{p J})\end{array}$ & $\begin{array}{c}\boldsymbol{T}_{\text {breakeven }} \\
(\mathbf{n s})\end{array}$ \\
\hline exu_alu & 429.5 & $15,022.5$ & 2.9 & 459.1 & 0.2 & 1.8 & 72.6 & 40.8 \\
exu_div & 3714.6 & $12,218.2$ & 30.4 & 3970.9 & 2.1 & 0.2 & 628.0 & 3924.0 \\
exu_ecl & 2319.3 & 6869.5 & 33.8 & 2479.4 & 2.3 & 0.1 & 392.1 & 4292.9 \\
exu_rml & 1729.2 & 4340.0 & 39.8 & 1848.5 & 2.7 & 0.4 & 733.1 & 1929.6 \\
ifu_dec & 277.5 & 4049.1 & 6.8 & 296.7 & 0.5 & 0.4 & 46.9 & 119.7 \\
ifu_fcl & 1785.6 & 5991.8 & 29.8 & 1908.8 & 2.1 & 0.5 & 301.9 & 616.6 \\
ffu_dp & 5466.6 & $13,722.1$ & 39.8 & 5843.8 & 2.7 & 1.3 & 924.2 & 716.1 \\
\hline
\end{tabular}

\subsection{Post-Fabrication Optimization: A Pulse Width Modulation}

We now describe a post-fabrication optimization strategy. The main idea is that a write pulse duration can be adaptively adjusted to reduce the write energy overhead for programs which have high fault tolerance. Because of a trade-off between energy and error probability of the propose NVFF, the write energy can be reduced by sacrificing error probability. This is true where each program has its unique fault tolerance even if the hardware design remains unchanged. In other words, some programs can tolerate more error so that we can use a shorter pulse duration for the programs to save more energy.

To implement this idea, we first examine the fault tolerance of programs to validate whether the fault tolerance varies over programs. Four programs - matrix multiply, sort, bzip2, and prime-are selected for this experiment. Gate-level simulations of the programs are performed on an OpenSPARC core using Synopsys VCS to inject faults and monitor the final outputs [28]. The fault injection process is based on a gate-level simulation that is halted at a randomly-determined cycle. The gate-level simulator extracts outputs of the combinational blocks and flip-flops for the cycle of interest. We inject faults (e.g., flipped value) on the flops based on the probabilities of their occurrence. After the injection, the analysis continues to the end of programs to determine whether the fault has been masked or a system failure has occurred. Outcomes from the fault injection are compared to a golden fault-free run. System failures by the fault injection are categorized as one of the following: detected unrecoverable error (DUE), Output match, silent data corruption (SDC), Hung, or Masked. We did this fault injection process for four programs. As shown in Figure 5, the most frequent category is Masked (above $90 \%$ of all cases). The second highest category is DUE, followed by SDC and Output match. The Hung case is not observed in the simulation. Among the four programs, bzip2 shows the lowest system failure rate, $1.2 \%$. The DUE is only $0.6 \%$, whereas the other programs are above $2.2 \%$. This clearly shows that bzip2 has better tolerance in this experiment; therefore, a shorter pulse duration can be used for the program to save more energy.

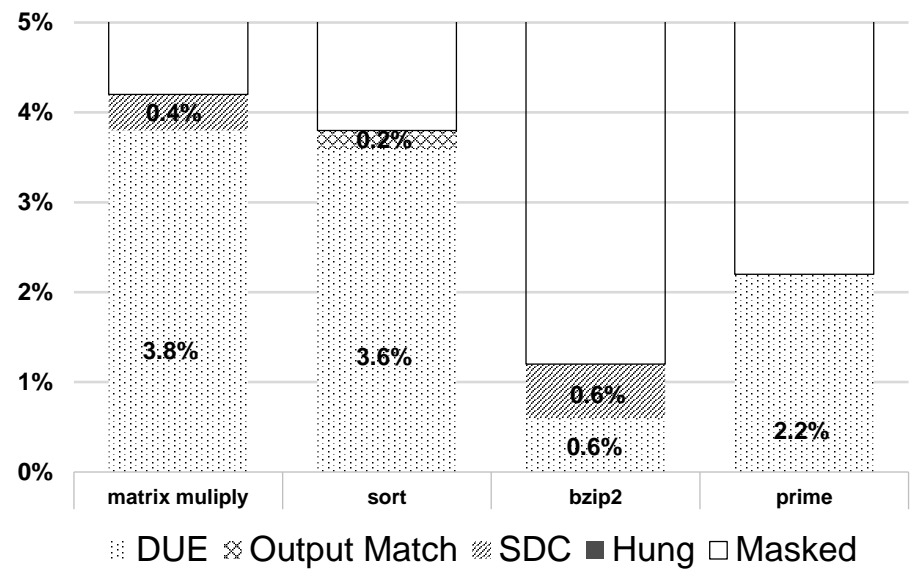

Figure 5. System failure results by fault injection. 
We also examine how much energy we can save by adjusting the pulse duration. Figure 6 shows the error rate and the expected energy of a flop at different pulse duration. The error probability is inversely proportional to the write pulse duration as Equation (1), and the expected energy is linearly increased by the pulse duration while the error rate is exponentially decreased. At 6.6 ns, a write error probability is $1.5 \times 10^{-13}$ and energy for a storing and restoring operations is $0.2 \mathrm{pJ}$. For short pulses such as $3.3 \mathrm{~ns}$, the computed error probability is increased to $3.9 \times 10^{-7}$ while the energy consumption is reduced by half. Because of such trade-off, the applied pulse duration for each program needs to be carefully selected based on the target error probability of the system even if the pulse duration modulation strategy maximizes energy savings of the proposed NVFF.

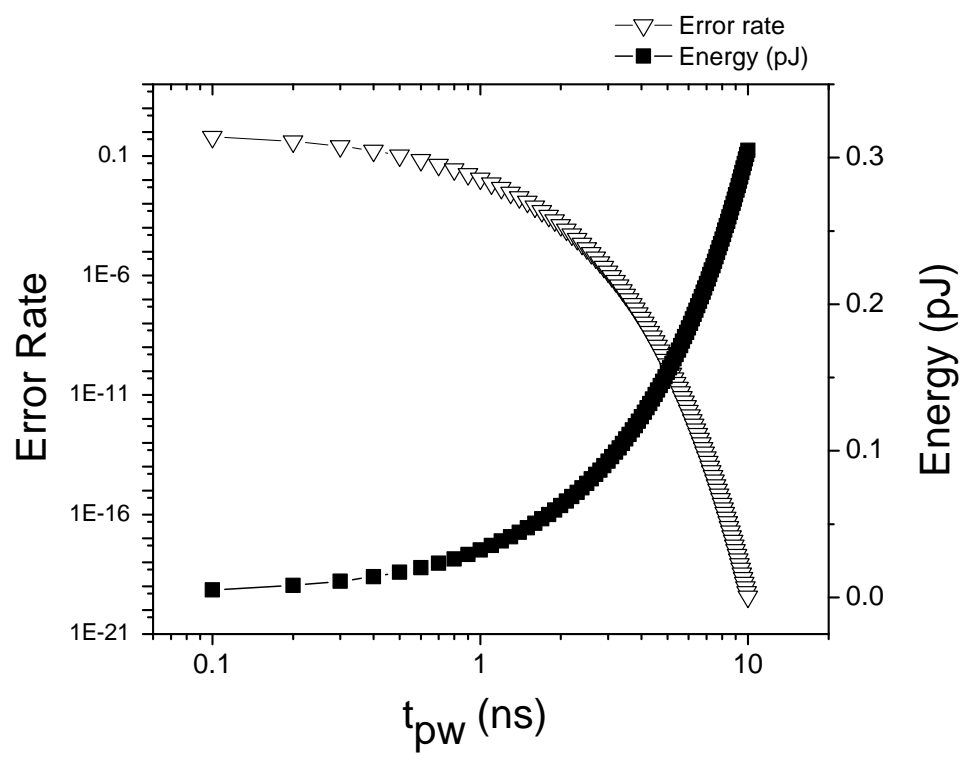

Figure 6. Error rate and the expected energy of the proposed NVFF at different write pulse duration.

In order to control the pulse duration, a control circuit is necessary. However, the area overhead per flop would be negligible because one circuit can control all FFs in a chip. In addition, the pulse duration is selected at software-level because the program information is needed.

\section{Conclusions}

In this paper, a novel area efficient NVFF is proposed. The relative area overhead is $6.9 \%$, and the proposed NVFF shows an improvement of nearly a factor of 4-23 in terms of area overhead compared to state-of-the-art NVFF designs. The write current for the restoring operation is reduced by $50 \%$ using the proposed current-reuse technique. To our knowledge, the proposed NVFF enables a fine-grained power gating without significant area overhead. Compared to the best previously known NVFFs, the proposed NVFF succeeds in reducing the area by $4.1 \times$ and the energy by $1.5 \times$.

Two optimization strategies for reducing area and energy overheads are also proposed: NVFF placement and pulse duration modulation strategies. We demonstrated the placement strategy on an OpenSPARC T1 core design. Analysis indicates that the placement on the ALU shows an improvement of nearly a factor of 2-18 in terms of area and energy compared to the other modules. We also demonstrated the fault tolerance variation over programs and the adaptive pulse duration strategy for the energy savings.

Author Contributions: J.P. contributed for methodology, validation, data curation, and writing-original draft preparation. Y.U.Y. contributed for writing - review and editing and supervision.

Funding: This work was supported by the National Program for Excellence in Software at Handong Global University (2017-0-00130) funded by the Ministry of Science and ICT.

Conflicts of Interest: The authors declare no conflict of interest. 


\section{Abbreviations}

The following abbreviations are used in this manuscript:

STT-MRAM spin torque transfer magnetic RAM

MTJ magnetic tunnel junction

FF flip flop

NVFF non-volatile flip flop

PTM predictive technology model

MUX multiplexer

EDK educational design kit

VCS verilog compiler and simulator

ALU arithmetic logic unit

DUE detected unrecoverable error

SDC silent data corruption

\section{References}

1. Shin, Y.; Seomun, J.; Choi, K.M.; Sakurai, T. Power gating: Circuits, design methodologies, and best practice for standard-cell VLSI designs. ACM Trans. Design Autom. Electron. Syst. 2010, 15, 28. [CrossRef]

2. Jeong, K.; Kahng, A.B.; Kang, S.; Rosing, T.S.; Strong, R. MAPG: Memory access power gating. In Proceedings of the Conference on Design, Automation and Test in Europe, Dresden, Germany, 12-16 March 2012; pp. 1054-1059.

3. Chiou, D.S.; Chen, S.H.; Chang, S.C.; Yeh, C. Timing driven power gating. In Proceedings of the 43rd Annual Design Automation Conference, San Francisco, CA, USA, 24-28 July 2006; pp. 121-124.

4. Sorin, D.J.; Martin, M.M.; Hill, M.D.; Wood, D.A. SafetyNet: Improving the availability of shared memory multiprocessors with global checkpoint/recovery. In Proceedings of the 29th Annual International Symposium on Computer Architecture, Anchorage, AK, USA, 25-29 May 2002; pp. 123-134.

5. Prvulovic, M.; Zhang, Z.; Torrellas, J. ReVive: Cost-effective architectural support for rollback recovery in shared-memory multiprocessors. In Proceedings of the 29th Annual International Symposium on Computer Architecture, Anchorage, AK, USA, 25-29 May 2002; pp. 111-122.

6. Sato, H.; Yamamoto, T.; Yamanouchi, M.; Ikeda, S.; Fukami, S.; Kinoshita, K.; Matsukura, F.; Kasai, N.; Ohno, $\mathrm{H}$. Comprehensive study of CoFeB-MgO magnetic tunnel junction characteristics with single-and double-interface scaling down to $1 X \mathrm{~nm}$. In Proceedings of the 2013 IEEE International Electron Devices Meeting, Washington, DC, USA, 9-11 December 2013; Volume 3, pp. 1-3.

7. Zhang, Y.; Zhao, W.; Lakys, Y.; Klein, J.; Kim, J.V.; Ravelosona, D.; Chappert, C. Compact Modeling of Perpendicular-Anisotropy CoFeB/MgO Magnetic Tunnel Junctions. IEEE Trans. Electron Devices 2012, 59, 819-826. [CrossRef]

8. Zhao, W.; Cao, Y. Predictive technology model for nano-CMOS design exploration. ACM J. Emerg. Technol. Comput. Syst. 2007, 3, 1. [CrossRef]

9. Worledge, D.; Hu, G.; Trouilloud, P.; Abraham, D.; Brown, S.; Gaidis, M.; Nowak, J.; O'Sullivan, E.; Robertazzi, R.; Sun, J.; et al. Switching distributions and write reliability of perpendicular spin torque MRAM. In Proceedings of the 2010 International Electron Devices Meeting, San Francisco, CA, USA, 6-8 December 2010.

10. Raychowdhury, A.; Somasekhar, D.; Karnik, T.; De, V. Design space and scalability exploration of 1t-1stt mtj memory arrays in the presence of variability and disturbances. In Proceedings of the 2009 IEEE International Electron Devices Meeting (IEDM), Baltimore, MD, USA, 7-9 December 2009.

11. Diao, Z.; Li, Z.; Wang, S.; Ding, Y.; Panchula, A.; Chen, E.; Wang, L.C.; Huai, Y. Spin-transfer torque switching in magnetic tunnel junctions and spin-transfer torque random access memory. J. Phys. Condens. Matter 2007, 19, 165209. [CrossRef]

12. Microsystem, S. OpenSPARC ${ }^{\mathrm{TM}} \mathrm{T} 1$ Microarchitecture Specification. 2009. Available online: https://www. oracle.com/technetwork/systems/opensparc/t1-01-opensparct1-micro-arch-1538959.html.

13. Dujmovic, J.J.; Dujmovic, I. Evolution and evaluation of SPEC benchmarks. ACM Sigmetrics Perform. Eval. Rev. 1998, 26, 2-9. [CrossRef] 
14. Yamamoto, S.; Shuto, Y.; Sugahara, S. Nonvolatile delay flip-flop using spin-transistor architecture with spin transfer torque MTJs for power-gating systems. Electron. Lett. 2011, 47, 1027-1029. [CrossRef]

15. Jung, Y.; Kim, J.; Ryu, K.; Kim, J.P.; Kang, S.H.; Jung, S.O. An MTJ-based non-volatile flip-flop for high-performance SoC. Int. J. Circuit Theory Appl. 2014, 42, 394-406. [CrossRef]

16. Ryu, K.; Kim, J.; Jung, J.; Kim, J.P.; Kang, S.H.; Jung, S.O. A magnetic tunnel junction based zero standby leakage current retention flip-flop. IEEE Trans. Very Large Scale Integr. (VLSI) Syst. 2012, 20, 2044-2053. [CrossRef]

17. Zhao, W.; Belhaire, E.; Chappert, C. Spin-mtj based non-volatile flip-flop. In Proceedings of the 2007 th IEEE Conference on Nanotechnology (IEEE NANO), Hong Kong, China, 2-5 August 2007; pp. $399-402$.

18. Suzuki, D.; Hanyu, T. Magnetic-tunnel-junction based low-energy nonvolatile flip-flop using an area-efficient self-terminated write driver. J. Appl. Phys. 2015, 117, 17B504. [CrossRef]

19. Kwon, K.W.; Choday, S.H.; Kim, Y.; Fong, X.; Park, S.P.; Roy, K. SHE-NVFF: Spin Hall effect-based nonvolatile flip-flop for power gating architecture. IEEE Electron Device Lett. 2014, 35, 488-490. [CrossRef]

20. Kimura, H.; Fuchikami, T.; Maramoto, K.; Fujimori, Y.; Izumi, S.; Kawaguchi, H.; Yoshimoto, M. A 2.4 pJ ferroelectric-based non-volatile flip-flop with 10-year data retention capability. In Proceedings of the 2014 IEEE Asian Solid-State Circuits Conference (A-SSCC), KaoHsiung, Taiwan, 10-12 November 2014; pp. 21-24.

21. Lo, C.P.; Chen, W.H.; Wang, Z.; Lee, A.; Hsu, K.H.; Su, F.; King, Y.C.; Lin, C.J.; Liu, Y.; Yang, H.; et al. A ReRAM-based single-NVM nonvolatile flip-flop with reduced stress-time and write-power against wide distribution in write-time by using self-write-termination scheme for nonvolatile processors in IoT era. In Proceedings of the 2016 IEEE International Electron Devices Meeting (IEDM), San Francisco, CA, USA, 3-7 December 2016.

22. Na, T.; Ryu, K.; Kim, J.; Kang, S.H.; Jung, S.O. A comparative study of STT-MTJ based non-volatile flip-flops. In Proceedings of the 2013 IEEE International Symposium on Circuits and Systems (ISCAS), Beijing, China, 19-23 May 2013; pp. 109-112.

23. Goldman, R.; Bartleson, K.; Wood, T.; Kranen, K.; Melikyan, V.; Babayan, E. 32/28nm Educational Design Kit: Capabilities, deployment and future. In Proceedings of the 2013 IEEE Asia Pacific Conference on Postgraduate Research in Microelectronics and Electronics (PrimeAsia), Visakhapatnam, India, 19-21 December 2013; pp. 284-288.

24. Design Compiler; User Guide, Synopsys, 2000. Available online: https://www.synopsys.com/ implementation-and-signoff/rtl-synthesis-test/design-compiler-graphical.html.

25. IC Compiler, User Guide, Synopsys, 2013. Available online: https://www.synopsys.com/implementationand-signoff/physical-implementation/ic-compiler.html.

26. PrimeTime, User Guide version c-2009.06. Synopsys, June 2009. Available online: https:/ / www.synopsys. $\mathrm{com} /$ content/dam/synopsys/implementation\&signoff/datasheets/primetime-ds.pdf.

27. Borkar, S. Design challenges of technology scaling. IEEE Micro 1999, 19, 23-29. [CrossRef]

28. Verilog Compiler Simulator Synopsys, 2004. Avaliable online: https://www.synopsys.com/verification/ simulation/vcs.html.

(C) 2019 by the authors. Licensee MDPI, Basel, Switzerland. This article is an open access article distributed under the terms and conditions of the Creative Commons Attribution (CC BY) license (http://creativecommons.org/licenses/by/4.0/). 